\title{
The future of deciphering personal genomes? The flies (and yeast and worms) still have it
}

\author{
Adam P Rosebrock ${ }^{* 1}$ and Amy A Caudy ${ }^{* 1,2}$
}

\section{Abstract \\ A report on the 13th International Conference on Systems Biology, held in Toronto, Ontario, Canada, 19-23 August 2012.}

\section{Introduction}

Sydney Brenner said in his 2002 Nobel lecture that we are 'drowning in a sea of data, starving for knowledge.' Drafts of the human genome sequence were barely a year old at the time, and even optimistic projections placed the $\$ 1,000$ genome beyond 2040. Just a decade after Brenner's lecture, technological revolutions in sequencing and protein analysis have changed the pace of genome biology. The reference genome has given way to individual patient sequences. Sydney's sea of data is becoming ever deeper.

New tools are necessary to extract biological meaning from these data. The diverse group of scientists gathered in systems biology is at the forefront of devising new ways to net knowledge out of a data sea that widens and deepens every day. The flood of data produced by modern 'omics technologies demands new methods that connect genotype to phenotypic consequence. With the $\$ 1,000$ genome now predicted by the end of 2012, we are faced with the challenge of interpreting raw sequence into health-relevant information.

Here, we cover some of the highlights of an inherently diverse meeting, focusing on the role of systems biology as relevant to genomic medicine (abstracts are freely available online: http://www.icsb2012toronto.com/schedule.php).

\section{Biological systems are dynamic; systems biologists are catching up}

Twenty-three pairs of chromosomes within a single cell give rise to hundreds of tissues with diverse functions,

\footnotetext{
*Correspondence: adam.rosebrock@utoronto.ca, amy.caudy@utoronto.ca 'Donnelly Centre for Cellular and Biomolecular Research, University of Toronto, 160 College St., Toronto, Ontario, Canada, M5S3E1

2Department of Molecular Genetics, University of Toronto, 160 College St., Toronto, Ontario, Canada, M5S3E1
}

morphologies and biochemical activities. The humble budding yeast is born, has a fruitful replicative life on a variety of foods, and eventually succumbs to senescence. Life consists of a series of changing processes that cannot be explained by a snapshot view; models that accurately represent biological processes must be built from data that capture these dynamics.

Advances in mass spectrometry have enabled examination of rare and modified proteins; modified proteins are turning out to be not rare at all. Kirti Sharma in Matthias Mann's lab (Max Planck Institute of Biochemistry, Martinsreid, Germany) claims that $75 \%$ of the proteins in cancer cells are present as phosphorylated forms, and that this is likely to be a vast underestimate, with current methods capturing only one-tenth of the phosphopeptides in the cell.

A major focus of systems biology is understanding the interaction between biomolecules. Genetic approaches, including systematic identification of synthetic lethal gene deletions (championed by Charley Boone at the University of Toronto, ON, Canada), are being supplemented by measurement of physical interactions. These efforts are focused on reducing the massive disconnect between genome sequence and physical function.

Many key proteins in the cell are interaction hubs and form complexes with many different partners. Interactions are frequently mutually exclusive or separated in space, time, or by cellular state. Anne-Claude Gingras (Samuel Lunenfeld Research Institute, Toronto, ON, Canada) and Andrew Emili (University of Toronto, Toronto, ON, Canada) are working to move from a simple static representation of protein complexes to an understanding of these dynamic interactions. Gingras is interrogating protein interactions by affinity purification of different components of a single complex. Emili has taken a global approach by analyzing the entire proteome following multiple orthogonal separation methods and identifying complexes by common separation patterns. Michael Washburn (Stowers Institute, Kansas City, MO, USA) has been using mass spectrometry to address questions once reserved for structural biology. Washburn has used affinity purification and mass spectrometry to measure protein interactions in partially disassociated 
complexes. Interactions that survive are likely to occur between directly contacting molecules.

Weak interactions are common and essential in biological systems. Mike Tyers (IRIC, Université de Montréal, QC, Canada) reminded listeners that without weak interactions, we would be stones. Tyers' work demonstrates that multiple weak sites provide a mechanism for buffering, redundancy and cooperative kinetic behavior. Disruption of individual weak-functioning residues can frequently be tolerated, confounding current mutationcounting sequencing efforts.

\section{Model organisms drive understanding of genotype to phenotype}

Studies in model organisms can answer questions that have proved to be difficult to study in man. Missing heritability is one of the biggest problems in human genome-wide association studies. Joshua Bloom (Princeton University, Princeton, NJ, USA) mapped dozens of quantitative trait loci in yeast. They were able to account for more than $90 \%$ of the heritability of these traits, suggesting that the failure to map quantitative traits in human populations results from the diversity of alleles and the influence of environment rather than a problem with the approach of mapping.

Enrolling more patients is a common way to increase the power of genome-wide association studies. Two talks at the meeting presented a systems-biology alternative. Chad Myers (University of Minnesota, Minneapolis, MN, USA) and Manuel Mattheisen (Brigham and Williams Hospital, Boston, MA, USA) demonstrated approaches that group genes prior to testing for significant variants. Both researchers used functional groupings of genes based largely on data from model systems. Model organisms continue to be a critical scaffold for understanding biology.

Ben Lehner (ICREA, Barcelona, Spain) highlighted how chance controls life. Caenorhabditis elegans harboring null alleles of Tbx-9, a transcription factor regulating development, have a fifty-fifty chance of death or completely normal life. Lehner's group has identified fluctuations in gene expression within this isogenic population that modulate this life and death coin-toss. In our world of Brownian motion and stochastic gene expression, deterministic sequence to phenotype analysis will remain the stuff of science fiction.

\section{'Personal genomes' are of more value to the community than the patient}

Geneticist Sewall Wright once said that natural variation is the worst kind of experiment to study. Wright, the father of genetic drift and the fitness landscape, had a prescient understanding of our predicament. Mike Snyder (Stanford University, Stanford, CA, USA) gave an update on the Snyderome, a longitudinal study of his transcriptome, proteome, metabolome and other physiological parameters in what may well be the most expensive case study in history. Next-generation sequencing has become Snyder's 'Ouiji board'. His risk allele for diabetes may have manifested itself in an episode of elevated blood glucose, which Snyder (possibly) corrected by a wholesale elimination of sweets. Fortunately for Snyder, none of his other disease risk alleles have been borne out or have required such radical life changes. Personal genome sequencing is here, but the interpretation is difficult, and as Snyder advises, not for worriers.

According to Chris Saunder (Memorial Sloan Kettering Cancer Center, New York, NY, USA), genome medicine is sequence-rich and phenotype-poor. Saunder remarked that the erudite molecular profiling of the Cancer Genome Atlas and related efforts frequently lacks accompanying data on phenotype, cautioning that careful and deep phenotyping is as important as sequencing itself. George Church (Harvard University, Cambridge, MA, USA) highlighted efforts coordinated through the Personal Genomics Project to provide such rich phenotypic information. This dataset of genomes coupled with phenotype will be useful in understanding the scope of variation, but Church cautioned that the diagnostic value of genomes is limited. Like a stethoscope, sequencing is just one source of health information.

Identification of causative alleles is particularly critical in cancer; cells that appear in relapse are often from a different or earlier lineage than cells in the original tumor. John Dick (Princess Margaret Hospital, Toronto, ON, Canada) described work to identify causative mutations in genomes using xenografts. As in relapsing patients, many of the tumors that grew in mice are genetically different from the primary tumor. Dick's work questions the value of a 'tumor genome' derived from bulk cells, emphasizing the need to identify rare cells that can form new tumors. Personalized medicine may well become cell-, not patient-, specific.

Dave Hill (CCSB, Boston, MA, USA) reminded attendees that many proteins are multifunctional. Patientspecific mutations will affect some or many of a diverse array of protein interaction partners. To move from sequence to diagnosis and prognosis, one needs to understand these complex interactions. Hill cautioned that early successes with Mendelian traits will not translate into rapid understanding of complex genetic interactions.

The most remarkable personal genome, though, was that of only half a human. Thijn Brummelkamp (Netherlands Cancer Institute, Amsterdam, The Netherlands) presented a series of functional genetic screens using a haploid somatic human cell line. Brummelkamp used traditional insertional mutagenesis to identify essential human genes, bringing classic tools of model organism research to human genetic analysis. 
In addition to new tools, systems biology requires new ways of training tomorrow's scientists

Radical advances in technology have driven systems biology in the past decade. George Church highlighted the phenomenal growth of sequencing technology, noting that 44 commercial sequencing technologies are now available or soon will be. New sequencing technologies will be cheaper, faster and more accurate, and will permit new types of analyses.

The diverse skills required by systems biology require development of a new kind of scientist. David Botstein (Princeton University, Princeton, NJ, USA) accepted an award for excellence in quantitative education for his creation of a new teaching paradigm. Botstein's view is that students languish in prerequisite general courses while thirsting for access to current research and educational specialization. Contrary to the status quo, Botstein envisions research and teaching as organically connected tasks. This integrated training approach is necessary to bridge the significant cultural gap between biologists, mathematicians and computer scientists. Students should be taught current scientific practice, not scientific history. If the acceleration in systems biology continues, merely keeping up with the present will be a monumental task.

Published: 27 September 2012

doi:10.1186/gm375

Cite this article as: Rosebrock AP, Caudy AA: The future of deciphering personal genomes? The flies (and yeast and worms) still have it. Genome Medicine 2012, 4:74 